\title{
Delay tolerant networking with data triage method based on emergent user policies for disaster information network system
}

\author{
Noriki Uchida $^{\mathrm{a}, *}$, Norihiro Kawamura ${ }^{\mathrm{b}}$, Goshi Sato ${ }^{\mathrm{c}}$ and Yoshitaka Shibata ${ }^{\mathrm{c}}$ \\ ${ }^{a}$ Department of Informational Society Studies, Saitama Institute of Technology, Fukaya, Japan \\ ${ }^{\mathrm{b}}$ TAC Engineering Co., LTD., Tsushidanishi, Morioka, Iwate, Japan \\ ${ }^{\mathrm{c}}$ Faculty of Software and Information Science, Iwate Prefectural University, Iwate, Japan
}

\begin{abstract}
When Disaster Information Network System is considered in local areas that were heavy damaged by the East Japan Great Earthquake in 2011, the resiliency of the network system is one of significant subjects for the restoration of the areas. DTN (Delay Tolerant Network) has been focused for the effective methods for such inoperable network circumstances. However, when DTN is applied for the local areas, there are some problems such as message delivery rate and latency because there are fewer roads, cars, and pedestrians than in urban areas. In this paper, we propose the Enhanced Media Coordinate System for its architecture, and Data Triage method by emergent user policies is introduced to improve the QoS in Disaster Information Network System in local areas. In the proposed method, every message is tagged with the priority levels by data types with considering emergent user policies, and the high priority messages are firstly duplicated to transmittable nodes. Then, the experimental results by the GIS map of a Japanese coastal town and the future studies are discussed.
\end{abstract}

Keywords: Disaster information system, delay tolerant network, QoS

\section{Introduction}

Over two years have already passed since the East Japan Great Earthquake in 2011, many damaged Japanese coastal cities still have many problems for the restoration. Especially, in the restoration plan about rebuilding the cities, many people still live in temporal residents and the reconstruction of these buildings are not so much in progress. Although there are political reasons for these late reconstructions, it is also necessary to consider about the disaster prevention against earthquake or tsunami in the future. Not only the location of residential areas is important, but also the emergent communication methods are important if there would be an ultra large scale disaster in the future. Therefore, when one considered about Disaster Information Network System in these cities, the resiliency of the network system became one of significant research subjects in those days.

So far, the authors have studied two kinds of approaches in order to provide the resiliency for Disaster Information Network System. One of the proposed methods is Never Die Network (NDN) [1,2] methods considered as a robustness of network connection, and it consists of multiple wireless network interfaces

\footnotetext{
${ }^{*}$ Corresponding author: Noriki Uchida, Department of Informational Society Studies, Saitama Institute of Technology, 1690 Fusaiji, Fukaya, Saitama 369-2093, Japan. E-mail: uchida@sit.ac.jp.
} 
including Satellite System and amateur radio system that are supposed to be strong for damages. Since the Satellite and the amateur radio networks have the QoS (Quality of Service) problems such as throughput or jitter, NDN cognitively selects the proper wireless links and routes by the extend AHP (Analytic Hierarchy Process) method and the AODV (Ad hoc On-Demand Distance Vector) with Min-Max AHP values. This method is suitable for the fixed station such as disaster headquarters or evacuation shelters. Second is Delay Tolerant Networking (DTN) methods considered as the quick reactivation under inoperable circumstances. However, the previous papers [3-5] pointed out the data transmission efficiency about DTN, and they proposed different types of DTN protocols such as the Spray and Wait, MaxProp, and PROPHET in order to improve the delivery rate and latency of transmission data. Moreover, when it is considered DTN in local cities that are severely damaged by the East Japan Great Earthquake, the efficiency of DTN is supposed to be worse than that of the urban area because the transmittable nodes such as cars or pedestrians are fewer. Therefore, the authors previously studied about the efficiency of DTN in these actual Japanese coastal areas [6], and then we proposed the additional functions such as cognitive wireless network methods [7,8] or directional antenna control methods [9] for the DTN based Disaster Information System. However, in case of the actual disaster, it is necessary to consider more efficient and simple equipment for the network reactivations, so different approaches of DTN routing was needed for the future studies.

Therefore, this paper proposes a novel DTN architecture with the Enhanced Media Coordinate System (EMCS) for DTN to realize dynamic parameters control by the nodes' environments, the network conditions, and the user policies under emergent situations. Besides, the proposed methods mainly consist of three additional functions for DTN such as Data Triage by user policies, Node Selection methods by its territory, and Dynamic FEC (Forward Error Correction) by population estimation method [10]. Especially, this paper newly deals with the efficiency of the previous DTN routings by the comparison with the middle city and the local city in Japanese coastal area by the extended experiments of our proposed Data Triage Method. The results show that the Data Triage Method is effective than other DTN protocols in local areas, and it is discussed for the future studies of DTN usages for Disaster Information Network System in local areas.

\section{Related works}

\subsection{Delay torelent networking}

DTN (Delay Tolerant Networking) is the approach of "challenged computer network" that provides interoperable communication where continuous end-to-end connectivity cannot be assumed [9]. Therefore, DTN generally takes into account "store-carry-forward" typed protocol for its routing. The basic typed DTN protocol is called the epidemic model because the spread models of virus can predict it. SIR model [10] is one of the epidemic DTN models that are used for the estimation of data delivery rates or the latency (delivery time). When " $S$ " is set to a susceptible population, " $I$ " is set to an infected population, and " $R$ " is set to a recovered population, the evolution of the epidemic is defined by the following equations:

$$
\begin{aligned}
& \frac{d S(t)}{d t}=-\beta S(t) I(t) \\
& \frac{d I(t)}{d t}=-\gamma I(t)+\beta S(t) I(t)
\end{aligned}
$$




$$
\frac{d S(t)}{d t}=-r I(t)
$$

In the Eqs (1) (2) (3), the parameter $\beta$ is the infection parameter and represents the rate of contacts between two individuals. The parameter $\gamma$ is the recovery rate and represents the rate in which infected individuals are recovered.

However, many previous studies [3-5] pointed out that the epidemic routing of DTN has some considerations to apply for the actual case of ad-hoc computer network. One is the limitation of a node's resources such as storage volumes, battery, and bandwidth. If a node is assumed as a cellular phone, the data volume is especially limited being only able to hold a certain volume of copies of messages. Also, the oldest data is abandoned in the epidemic routing if the capacity of a node's volume becomes full. Second is the delivery rate. There are many considerable subjects to carry the messages such as the number of encounters, the delivery distance, the network condition, and the node's movements. Third is delivered time (latency) from a sender node to a destination node. Since the mobile nodes carry messages, DTN is not suitable for the real-time contents. Also, some other factors such as encounters, distance, and network conditions are supposed to affect the latency. The difficulty of the delivery rate and latency can be also estimated by the SIR model.

Therefore, there are some different approaches of DTN routing such as Spray and Wait, MaxProp, and PROPHET in order to improve QoS of DTN. Spray and Wait [3] attempts to gain the resource efficiency by setting an upper limit of copies per message in the network. It is supposed to be effective for the low resource environments such as a low storage of mobile nodes. MaxProp [4] is the flooding-based routing as well as the epidemic routing, but it determines the message priority to be transmitted first and to be dropped first. The priorities are based on the path likelihoods to peers according to historical data and also on several complementary mechanisms, including acknowledgments, a head-start for new packets, and lists of previous intermediaries. PROPHET (The Probabilistic Routing Protocol using History of Encounters and Transitivity) [5] is a probabilistic routing protocol by using history of node encounters and transitivity to enhance performance over previously existing protocols.

However, even if these approaches are applied for the Disaster Information Network System in local areas or severely damaged areas, there should be more considerations to improve the delivery rate or the latency. That is because the transportation mainly consists of cars, and pedestrians are rare. Besides, available roads and cars are supposed to be fewer than in urban areas. In fact, our previous papers [6,7] evaluated the DTN for the Disaster Information System in the local area, and reported that the delivery rate is about $80 \%$, and that the latency is more than 2,000 seconds in consumption with DTN in the local town in Northern Japanese coastal area. Besides, in case of emergent use, there are some important messages related to human life that should be never lost. Therefore, DTN under disasters needs additional functions to improve especially the delivery rate.

\subsection{Disaster information network system}

For Disaster Information Network System, the robustness and quick reactivation of communication network are supposed to be important. Therefore, the authors have studied two kinds of approaches in order to provide the resiliency for Disaster Information Network System.

One of the proposed methods is Never Die Network (NDN) [1,2] methods considered as a robustness of network connection, and it consists of multiple wireless network interfaces including Satellite System and amateur radio system that are supposed to be strong for damages. Since the Satellite and the amateur radio networks have the QoS (Quality of Service) problems such as throughput or jitter, NDN cognitively 


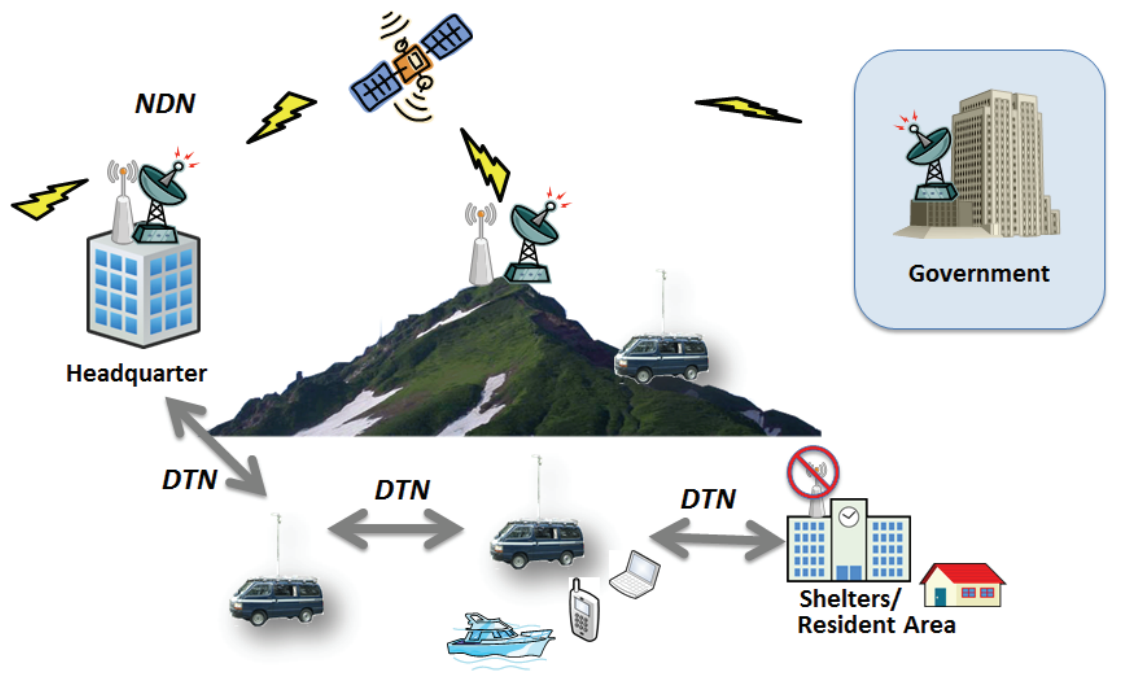

Fig. 1. The proposed disaster information network system.

selects the proper wireless links and routes by the extend AHP (Analytic Hierarchy Process) method and the AODV (Ad hoc On-Demand Distance Vector) with Min-Max AHP values. This method is suitable for the fixed station such as disaster headquarters or evacuation shelters.

Second is Delay Tolerant Networking (DTN) methods considered as the quick reactivation under inoperable circumstances. This method is suitable for the mobile station such as vehicle to vehicle communication or mobile terminal's communication. However, as previously mentioned, there are some considerable difficulties such as the delivery rate and latency for the actual usages. Especially, in case of local areas, the efficiency of DTN is supposed to be worse than that of the urban area because the transmittable nodes such as cars or pedestrians are fewer. Therefore, the authors previously proposed the additional functions such as cognitive wireless network methods $[7,8]$ or directional antenna control methods [9] for the DTN based Disaster Information System. As the results, the directional antenna control methods works effectively by the experiments. However, for the proposed antenna controls, the adjust equipment will be needed for the directional antenna controls, and it is consider to use more simple devices especially under the emergent situations.

\section{System architecture}

Our proposed Disaster Information Network System is shown in Fig. 1.

In the proposed system, the fixed stations such as headquarter and evacuation shelters are connected by NDN as previously mentioned, and the mobile nodes such as wireless cars, cellular phones, and mobile PCs are connected by the proposed DTN with Data Triage methods. Therefore, even if some shelters are isolated by the damages, disaster information will be carried by these DTN mobile nodes.

For the proposed system, this paper proposes the Data Triage methods by user policies for improving the efficiency of DTN routing, and we also propose the novel DTN architecture with the Enhanced Media Coordinate System (EMCS) for DTN to realize dynamic parameters control by the nodes' environments, the network conditions, and the user policies under emergent situations.

The architecture of DTN is generally discussed by Bundle Layer, Convergence Layer, and Custody Transfer that are introduced by RFC5050 and RFC5326 [11,12]. However, most of previous studies 
Table 1

Functions of layers and plane of EMCS

\begin{tabular}{ll}
\hline $\begin{array}{l}\text { Synchronization } \\
\text { layer }\end{array}$ & $\begin{array}{l}\text { It performs inter/intra message synchronizations in between DTN messages. } \\
\begin{array}{l}\text { Data transform } \\
\text { layer }\end{array}\end{array}$ \\
$\begin{array}{l}\text { It gives additional data of Data Triage and Node Selection for messages. } \\
\begin{array}{l}\text { Stream manage- } \\
\text { ment plane }\end{array}\end{array}$ & $\begin{array}{l}\text { It perform data error detection and correlation by Dynamic FEC. } \\
\text { The most suitable QoS parameter values on each protocol values on each protocol layer are deter- } \\
\text { mined according to the user's QoS requirements, node environments, and available computing and } \\
\text { network resources. }\end{array}$ \\
$\begin{array}{l}\text { QoS maintenance } \\
\text { plane }\end{array}$ & $\begin{array}{l}\text { It observes the User Plane and send/receive QoS parameters about data transmission. } \\
\text { User plane }\end{array}$ \\
$\begin{array}{l}\text { It performs message data transmission } \\
\text { Control plane }\end{array}$ & It performs the negotiation and resource allotment for Data Triage and Node Selection \\
\hline
\end{tabular}

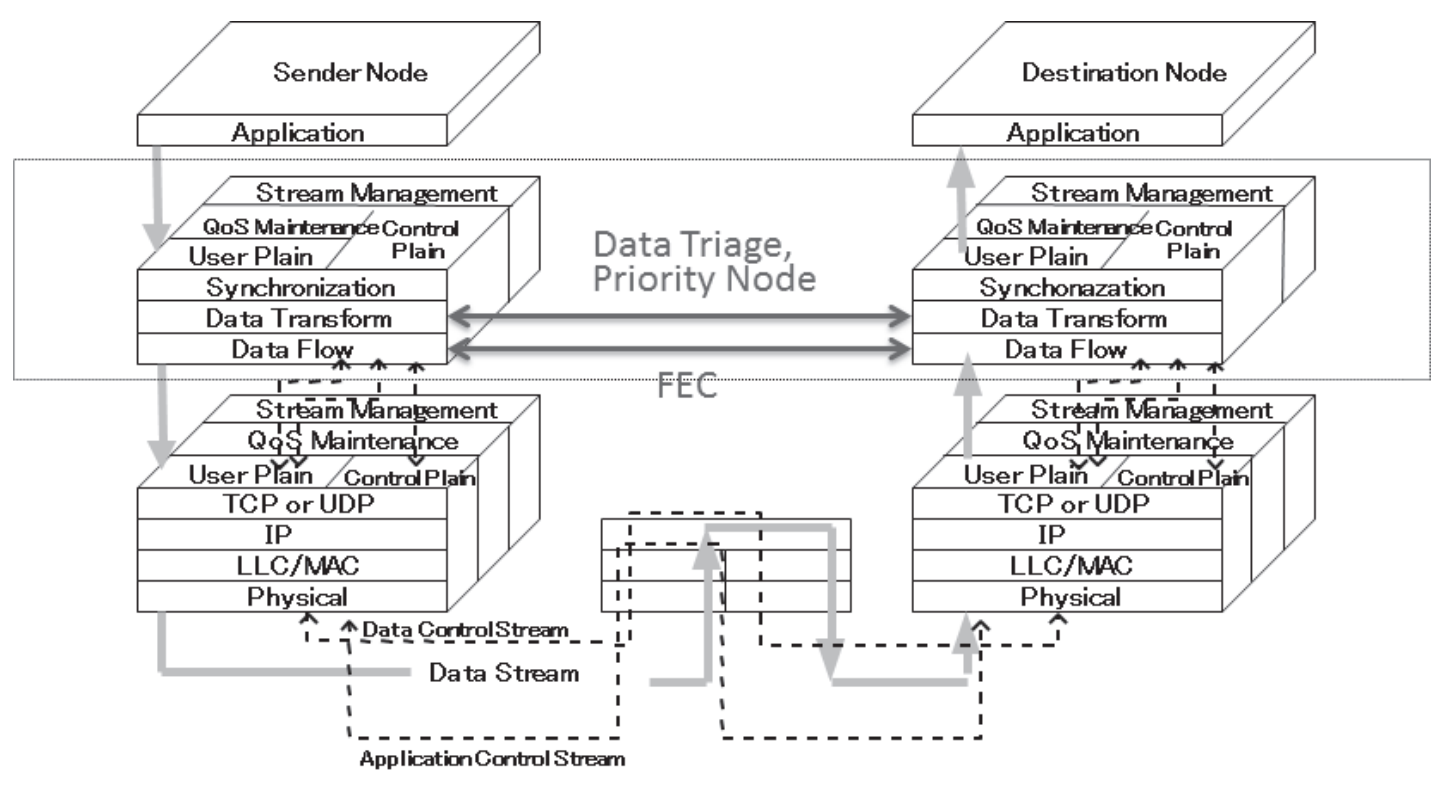

Fig. 2. The enhanced media coordinate system for DTN (EMCS).

such as MaxProp or PROPHET generally consider the nodes' environments or the network conditions for their proposed routings, and it is not suitable to realize them only by the previous DTN architecture. Therefore, as shown in Fig. 2, the proposed EMCS take into account for additional DTN functions by the various environments.

The media coordination system (MCS) is originally the architecture that is considered for the video communication system based on the peer-to-peer model [13], and it consists of three layers, including the synchronization layer, the data transform layer, and the media flow layer between the application layer and the transport layer in the OSI reference model to realize end-to-end QoS guarantee. Also, MCS is furthermore vertically divided into four planes such as the user plain, the QoS maintenance plain, the control plain, and the stream management plane by referring ATM architecture and adopted QoS-A model of Lancaster University [14].

In the proposed EMCS, each layer and plane are enhanced and works as the following Table 1. 


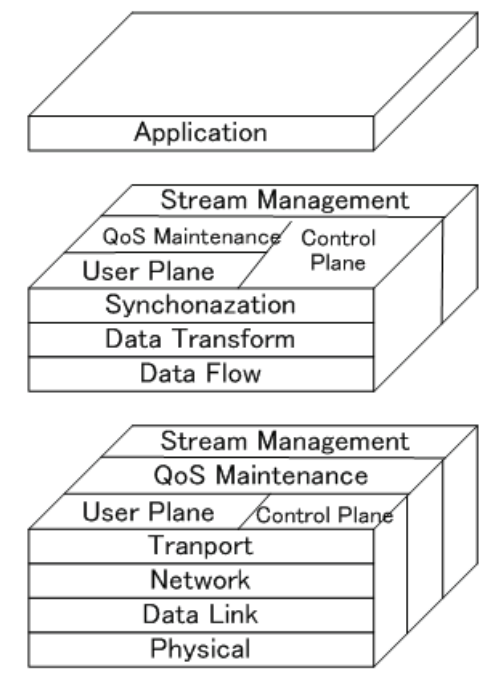

Fig. 3. The Proposed Functions by EMCS.

\section{Data triage}

With EMCS, we propose three additional functions for DTN such as Data Triage by user policies, Node Selection methods by Area of Interest, and Dynamic FEC (Forward Error Correction) by population estimation method. As shown in Fig. 3, Data Triage and Node Selection are held in Data Transform Layer among/between sender and receiver nodes. QoS parameters observed by each layers are transmitted by Control Plane toward each layers, and QoS Management Plane decides the weight of these functions. Then, Stream Management Plane controls the data transmission through User Plane. Dynamic FEC is also held in Data Flow Layer as the same way by these planes.

This paper especially deals with Data Triage methods for improving QoS of DTN. In order to decide message priority under emergent situations, it is necessary to consider what kinds of information will be needed. According to our previous study [15], the relationship between required information and time can be argued as the Fig. 4.

Before the disaster, if there is a rumor or news about upcoming disaster, the information of disaster prevention and evacuation shelter comes to be interested. These data are mainly consisted of text or pictures, and so data volume is not so much large and less important. However, if there is a disaster, the disaster information such as evacuation shelter, life safety, and damages becomes extremely important. This information is mainly consisted of text data, but whole data should be transmitted to the destination node. Then, evacuators come to need traffic information and relief supplies, and data volume becomes higher because they are consisted of text, map, and VoIP data. After about 2 weeks form the disaster, the information of lifeline and public service will be necessary, and data volumes will be increased since it is consisted of video data.

Therefore, in this paper, text data of rescue, evacuation, and safety status information are marked as the most important. Then, VoIP data is marked as moderately important, and video content data is less important for the Data Triage. After marked each message data by the user policies as mentioned before, the order of cashed messages are rearranged by the priority as shown in Fig. 5.

The current DTN stores messages by the chronological order, and the most recent message is delivered to transmittable nodes. On the other hand, the proposed Data Triage method stores messages as the user 


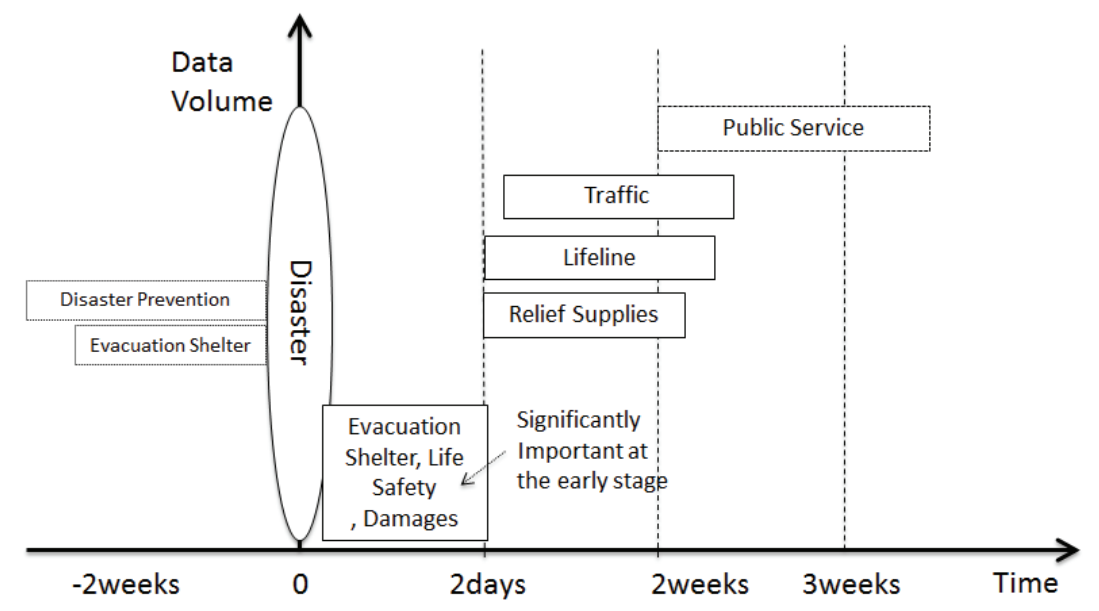

Fig. 4. The relationship between required information and time.

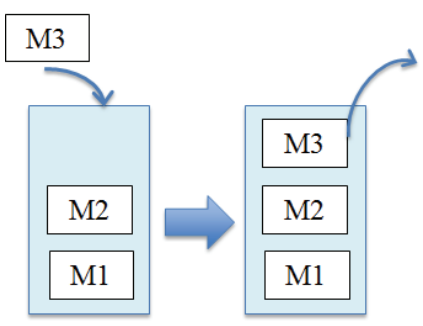

Current DTN

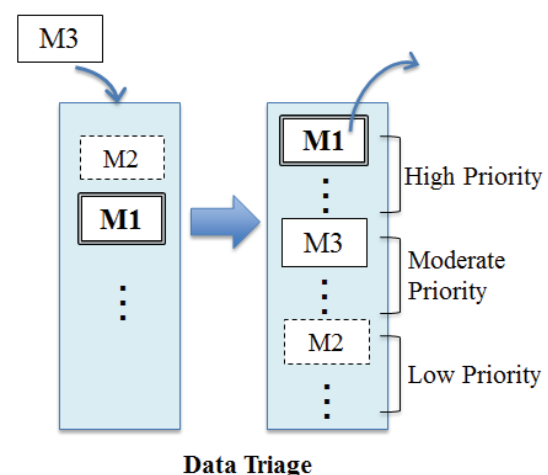

Data Triage

Fig. 5. Message order in cache by data triage.

policies, and the most significant message is delivered first. For example, in Fig. 5, if M1 is the high priority, M2 is the moderate, and M3 is the low, these cached messages would be rearranged as M1, M3, M2. Then, M1 is delivered faster than others.

\section{Experiments}

To evaluate the proposed Data Triage method, the computational simulations were held by using GIS map data. In the experiments, two Japanese cities are used in order to evaluate the effectiveness under local area's environments. One is Taro in Iwate prefecture that the population in 2013 was 1357, and the area is $101.15 \mathrm{~km}^{2}$ [16]. Another is Kamaishi in Iwate prefecture that the population in 2013 was 36381 , and the area is $441.42 \mathrm{~km}^{2}$ [17].

The ONE (The Opportunistic Network Environment Simulator) $[18,19]$ is used for the simulation. Also, as shown in Fig. 6, module modifications such as the internal routing logic, the GIS map, and connectivity data were implemented in order to realize the proposed methods. 
Table 2

Simulation senarios

\begin{tabular}{ll}
\hline Fixed wireless station & Taro: Seven buildings (City Hall and evacuation shelter designed by city) (IEEE802.11b). \\
& Kamaishi: Twenty buildings (City Hall and evacuation shelter designed by city) (IEEE802.11b). \\
Mobile modes & S-200 Wireless cars (IEEE802.11b). \\
& ShortestPath Model \\
& Speed 10-50 km/h \\
& (1) Epidemic, Spray and Wait, MaxProp, prophet in Taro \\
DTN routing & (2) Epidemic, MaxProp, and Data Triage in local city \\
& (3) Epidemic, MaxProp, and Data Triage in middle city \\
Wireless conditions & IEEE802.11b (11 Mbps, $100 \mathrm{~m}, 2.4 \mathrm{GHz})$ \\
& Non-directional Antenna \\
& RTT (43200 sec) \\
& $0.5-1.0$ MB data are created in every 50-60 seconds. \\
Data & Taro: Data are carried from Taro City Hall to Green Peer Taro. \\
& Kamaishi: Data are carried from Kosano Community Hall to Kamaishi Jr. school. \\
& In Data Triage, three types (high, middle, low) priority messages are generated randomly. \\
& 5 seconds duration is needed before data sending in order to such as PWD identification and \\
Network connection & DHCP. \\
Nodes & 2 GB storage. \\
Duration & 3 hours (10800 seconds). \\
\hline
\end{tabular}

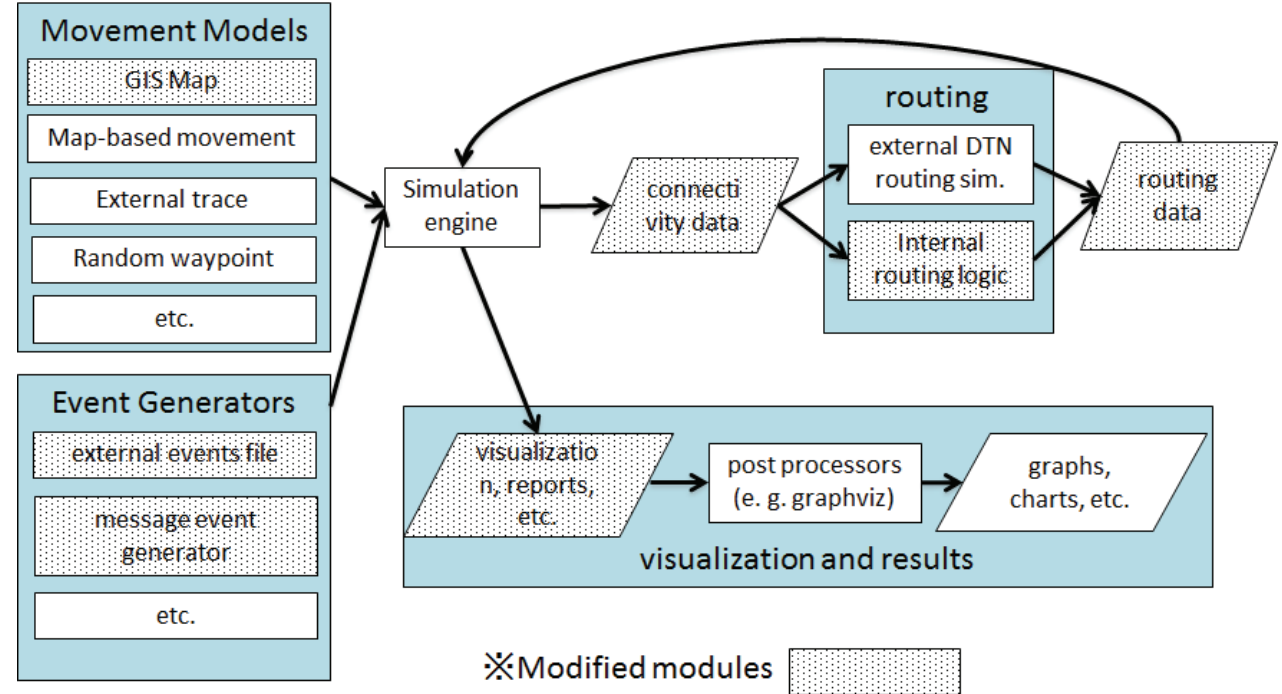

Fig. 6. The simulation map in the experiments.

In GIS map, the seven evacuation shelters, which are designated by the town, were located in the map of Taro, and mobile nodes assumed as wireless cars were also set in it. Also, 20 evacuation shelters are set in Kamashi. Table 2 indicates more details of simulation scenarios in these experiments.

First of all, to evaluate the efficiency of DTN in local are, the previous studied DTN routing, that is Epidemic, Spray and Wait, MaxProp, and PROPHET routing, was experimented by Taro town. As mentioned before, Taro is typical local area that has small population, and it is supposed to suitable for the experiment of DTN routing in local areas. Figure 7 shows the results of the delivery rate by the number of mobile nodes in Taro, and Fig. 8 shows that of the latency. 


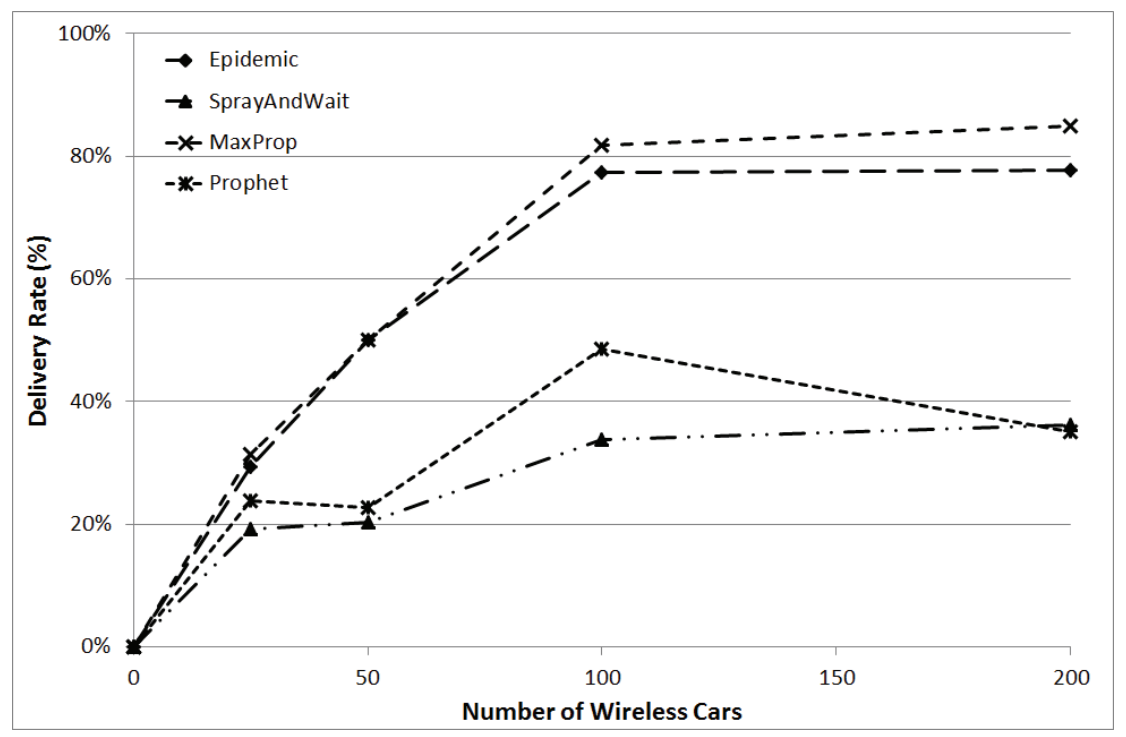

Fig. 7. The experimental results of the delivery rate and the number of wireless cars.

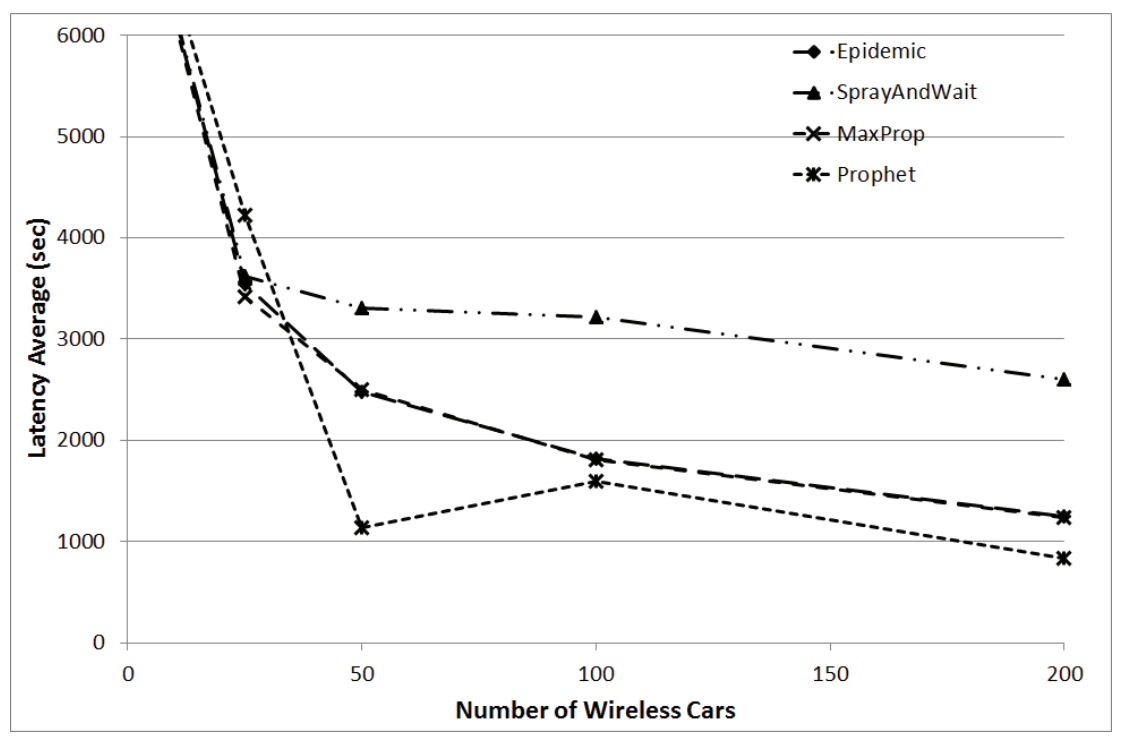

Fig. 8. The experimental results of the latency and the number of wireless cars.

According to Fig. 7, the graph of every DTN routing demonstrates the sharp rise by 50 or 100 mobile nodes, and then the probability is kept constant at around $40 \%$ or $80 \%$. The result is supposed to match the mathematical epidemic models of Eqs (1)-(3) as the previously mentioned. When these DTN routings are compared, Epidemic and MaxProp routings shows better results than others. That is because it is considered that the experimented nodes have enough storages as $2 \mathrm{~GB}$, and so message priority typed methods show better results than the cached efficient typed or path priority typed routing methods.

Figure 8 indicates the Spray and Wait routing shows better latency than others, and other routings show almost same latency. However, in case of disaster, some messages are significantly important to 


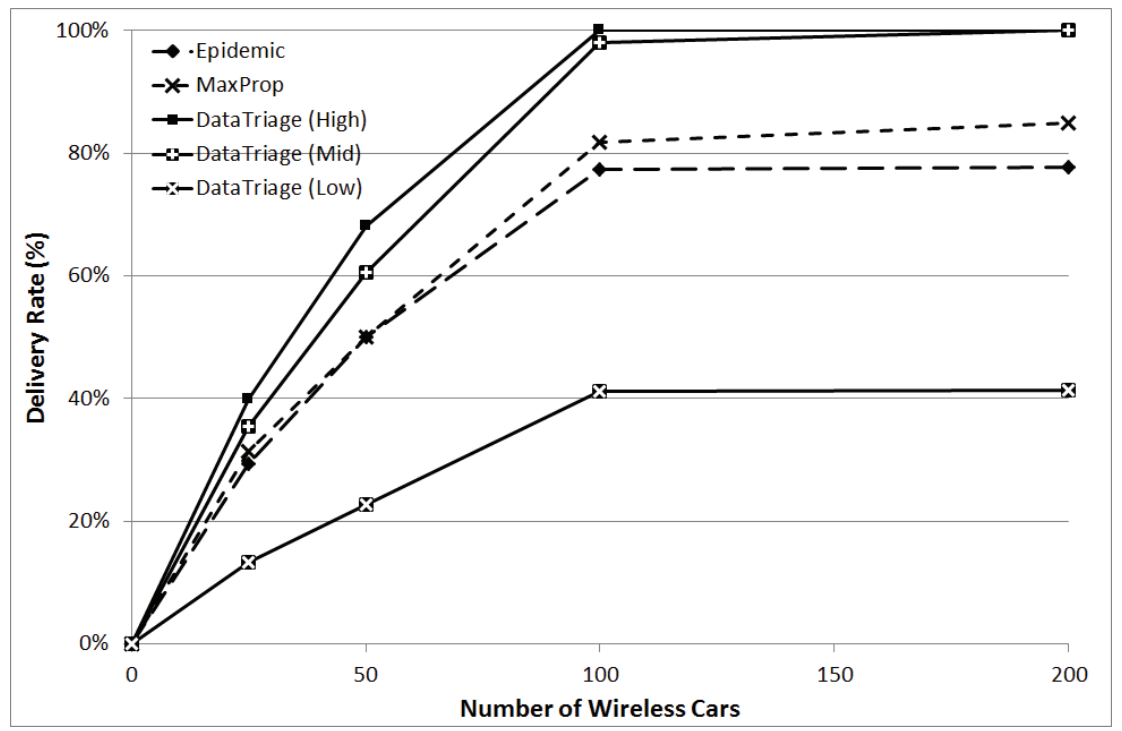

Fig. 9. The delivery rate by data triage method in Taro.

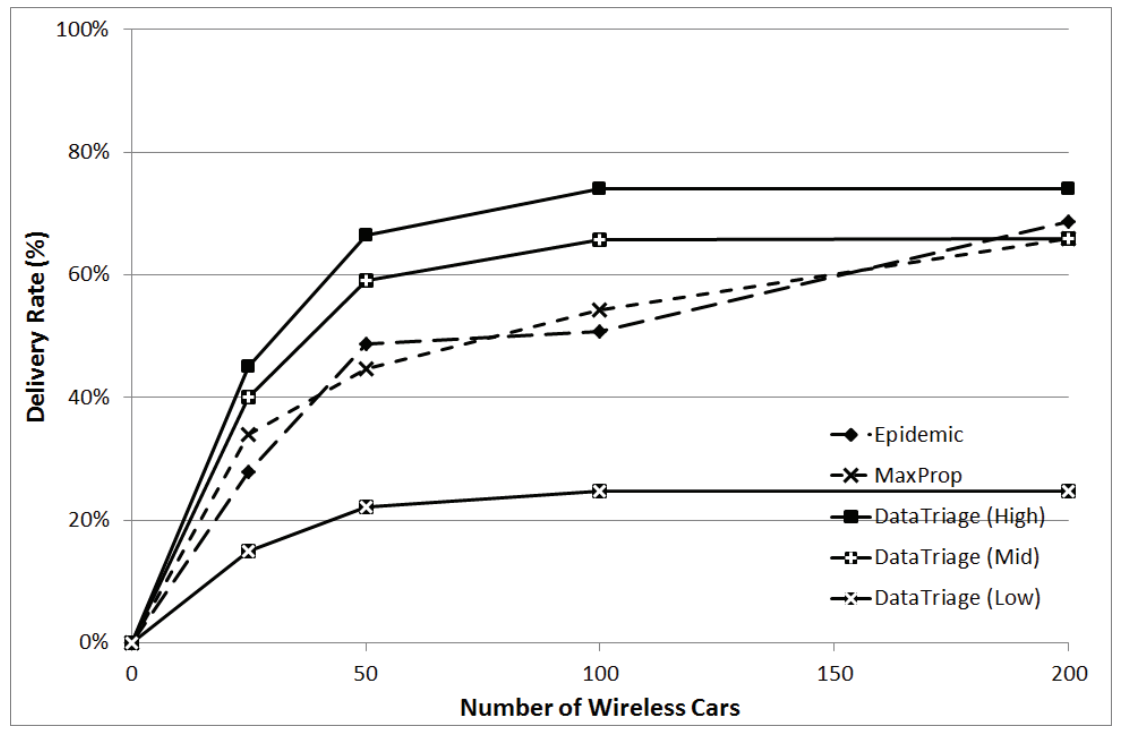

Fig. 10. The delivery rate of by data triage method in Kamaishi.

deliver because these messages are related to rescue or life. Therefore, it is considered to focus on the delivery rate more than the latency in such a situation, and epidemic and MaxProp routings are supposed to be suitable for Disaster Information Network System in local areas. Secondly, the experiments of the proposed Data Triage method are held in comparison with local areas and middle size area. To evaluate the efficiency of the proposed method, Data Triage, epidemic, and MaxProp routings are compared by the GIS map of Taro and Kamashi. Figure 9 indicates the results by Taro, and Fig. 10 indicates that of Kamaishi.

In these experiments by Data Triage, every message is randomly tagged by three types of priority that 


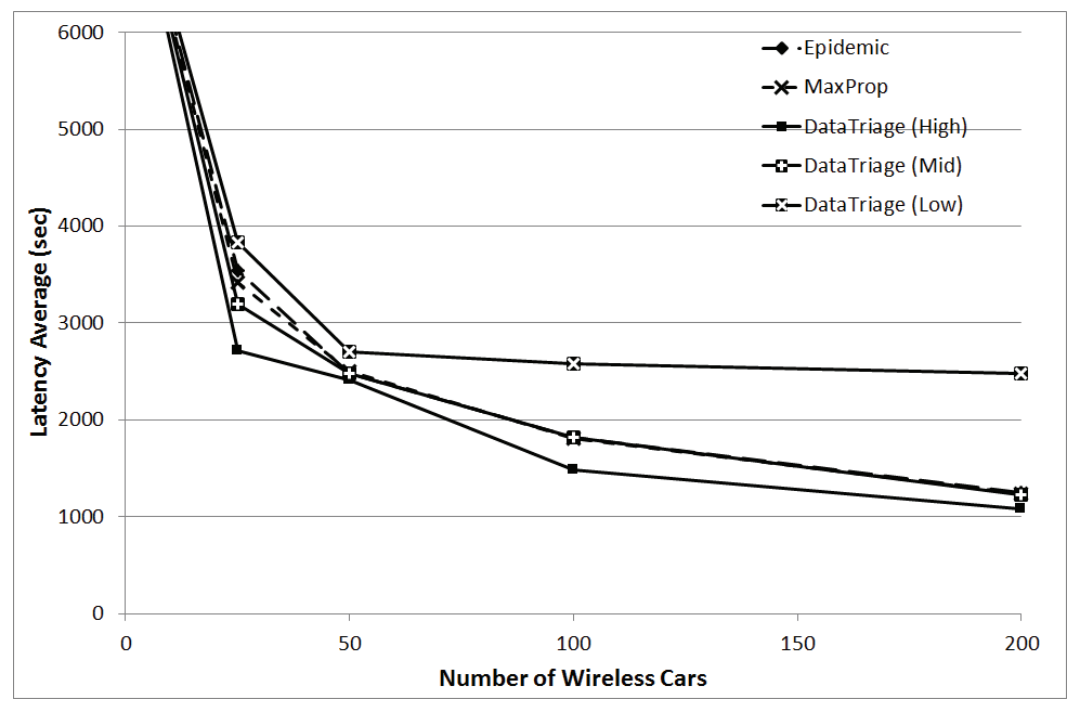

Fig. 11. The latency by data triage method in Taro.

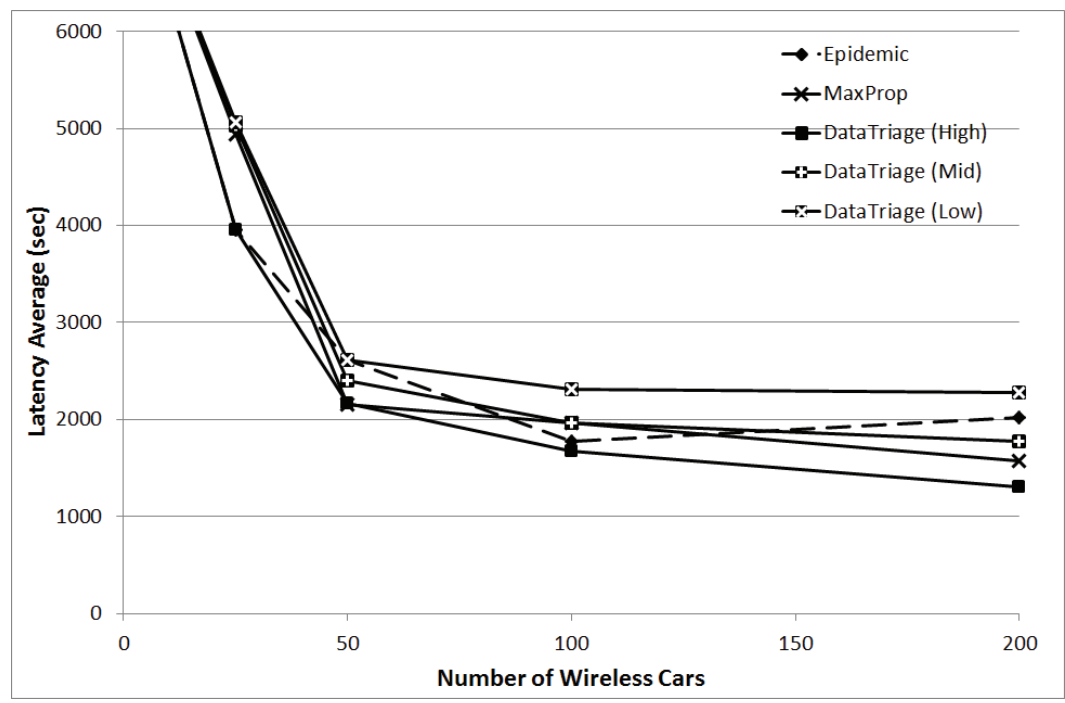

Fig. 12. The latency by data triage method in Kamaishi.

are high, middle, and low priority, and these three types of delivery rate are compared with Epidemic and MaxProp routings. According to Fig. 9, the high and middle priority tagged messages are superior than others, while the low priority messages are kept constant at around 40 with more than 100 mobile nodes. Thus, our proposed methods are supposed to be effective in local areas, and high priority messages can be delivered for the destination disaster headquarter or evacuation shelter effectively. Figure 10 indicates the results under the middle sized town. As the same as Fig. 9, high priority messages by Data Triage method shows better results than others, although the delivery rate is around $80 \%$ since the experiment area was higher. Thus, it is considered that our proposed methods can be also effective under the middle sized area. 
At last, the latency of the experiments shows in Figs 11 and 12. As the same as the results of the delivery rate, the latency in Taro and Kamaishi indicate the proposed methods are effective.

\section{Conclusions and future study}

When Disaster Information Network System is considered in local areas that were heavy damaged by the East Japan Great Earthquake, the resiliency of the network system became one of significant subjects for the restoration of the areas. That is because, if there is a large scale disaster, the disconnection or congestion of communication network might cause the lack of important information such as rescues, evacuation, and one's safety information. DTN is considered to be a possible method to transmit data in interoperable communication. However, if DTN is introduced for a Disaster Information Network System, the delivery rate and latency would become significant problems for the actual usage.

Therefore, this paper proposes a novel DTN architecture with the Enhanced Media Coordinate System (EMCS) for DTN to realize dynamic parameters control by the nodes' environments, the network conditions, and the user policies under emergent situations. Then, this paper proposed the novel Data Triage by emergent user policies, and the effectiveness is evaluated by the experiments that assumed to be used in local areas and middle size areas. The results indicate that the proposed Data Triage methods work effectively.

However, the delivery rate of high priority messages with the proposed Data Triage methods should be more improved for Disaster Information Network System, and so we are planning to additional functions for the Data Triage messages for the future studies. These are Node Selection methods by Area of Interest (AoI), and Dynamic FEC (Forward Error Correction) by population estimation method, and now we are studying for these functions for the future studies.

\section{Acknowledgments}

This paper is an extended version of the work originally presented at the 7th International Conference on Broadband and Wireless Computing, Communication and Applications (BWCCA2013), Compiegne, France, October 28-30, 2013. The authors also thank TAC Engineering Co., LTD. and Asia Air Survey Co., LTD. for the provision of GIS map data and the advice about the GIS technological operations.

\section{References}

[1] N. Uchida, K. Takahata, Y. Shibata and N. Shiratori, Never Die Network Based on Cognitive Wireless Network and Satellite System for Large Scale Disaster, Journal of Wireless Mobile Networks, Ubiquitous Computing, and Dependable Applications (JoWUA) 3(3) (Sep. 2012), 74-93.

[2] N. Uchida, K. Takahata, Y. Shibata and N. Shiratori, User Policy Based Link and Route Selection Methods for Never Die Network, Sixth International Conference on Broadband and Wireless Computing, Communication and Applications (BWCCA2011), Oct. 2011.

[3] T. Spyropoulos, K. Psounis and C.S. Raghavendra, Spray and Wait: An Efficient Routing Scheme for Intermittently Connected Mobile Networks, WDTN '05 Proceedings of the 2005 ACM SIGCOMM workshop on Delay-tolerant networking, 2005, pp. 252-259.

[4] J. Burgess, B. Gallagher, D. Jensen and B. Levine, MaxProp: Routing for Vehicle-Based Disruption-Tolerant Networks, INFOCOM 2006. 25th IEEE International Conference on Computer Communications. Proceedings, April 2006, pp. 111 .

[5] A. Lindgren, A. Doria and O. Scheln, Probabilistic routing inintermittently connected networks, ACM SIGMOBILE Mobile Computing and Communications Review 7(3) (July 2003), 19-20. 
[6] N. Uchida, N. Kawamura, N. Williams, K. Takahata and Y. Shibata, Proposal of Delay Tolerant Network with Cognitive Wireless Network for Disaster Information Network System, The 5th International Workshop on Disaster and Emergency Information Network Systems (IWDENS2013), Mar. 2013, pp. 249-254.

[7] N. Uchida, N. Kawamura and Y. Shibata, Delay Tolerant Networks on Vehicle-to-Vehicle Cognitive Wireless Communication with Satellite System for Disaster Information System in a Coastal City, IT CoNvergence PRActice (INPRA), ISSN: 2288-0860 (Online), Volume 1, Issue 1, March 2013, pp. 53-66.

[8] N. Uchida, N. Kawamura and Y. Shibata, Evaluation of Cognitive Wireless based Delay Tolerant Network for Disaster Information System in a Rural Area, The Seventh International Conference on Complex, Intelligent, and Software Intensive Systems (CISIS20123), July 2013, pp. 1-7.

[9] N. Uchida, N. Kawamura and Y. Shibata, Delay Tolerant Network with Directional Anntenna Control for Disaster Information System, The 16th International Conference on Network-Based Information Systems (NBIS2013), up to coming, Sep. 2013.

[10] N. Uchida, N. Kawamura, Y. Shibata and N. Shiratori, Proposal of Data Triage Methods for Disaster Information Network System based on Delay Tolerant Networking, The 7th International Conference on Broadband and Wireless Computing, Communication and Applications (BWCCA2013), up to coming, Oct. 2013.

[11] K. Scott and S. Burleigh, Bundle protocol specification, IETF RFC 5050, Nov. 2007.

[12] M. Ramadas, S. Burleigh and S. Farrell, Licklider transmission protocol-specification, IETF RFC 5326, Sept. 2008.

[13] K. Hashimoto, T. Chinen, J. Sato and Y. Shibata, Packet and Frame Rate Control Methods for Compressed Video Transmission, Special Issue on Multimedia Distributed and Cooperative Computing, IPSJ Journal 30(2) (1998), 337-347.

[14] A. Campell, G. Coulson and D. Hutchson, A Quality of Service Architecture, ACM SIGCOM Computer Communication Review 24(2) (1995), 1-27.

[15] D. Nakamura, N. Uchida, H. Asahi, K. Takahata, K. Hashimoto and Y. Shibata, Wide Area Disaster Information Network and Its Resource Management System, The 17th International Conference on Advanced Information Networking and Applications (AINA 2003), 2003, pp. 146-149.

[16] Miyako City, http://www.city.miyako.iwate.jp/cb/hpc/Article-1348-2716.html.

[17] Kamaishi City, http://www.city.kamaishi.iwate.jp/index.cfm / 10,22134,82,html.

[18] The ONE is a simulation, http://www.netlab.tkk.fi/tutkimus/dtn/theone/.

[19] A. Keranen, Opportunistic Network Environment simulator, Special Assignment, Helsinki University of Technology, Department of Communications and Networking, May 2008. 

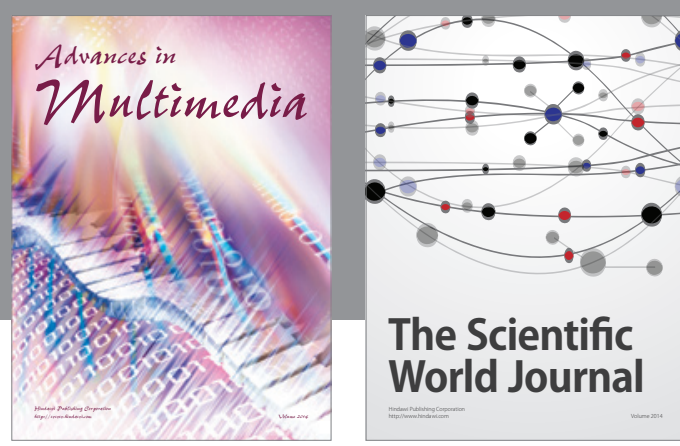

The Scientific World Journal
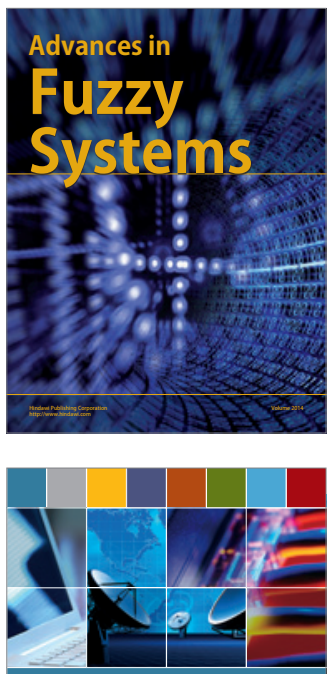

Computer Networks and Communications
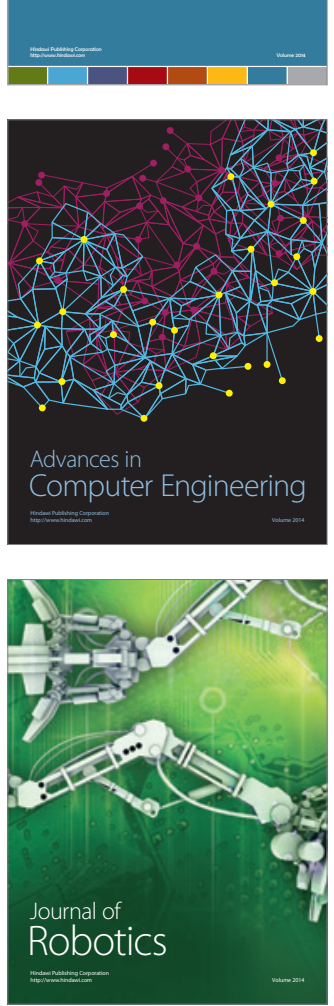
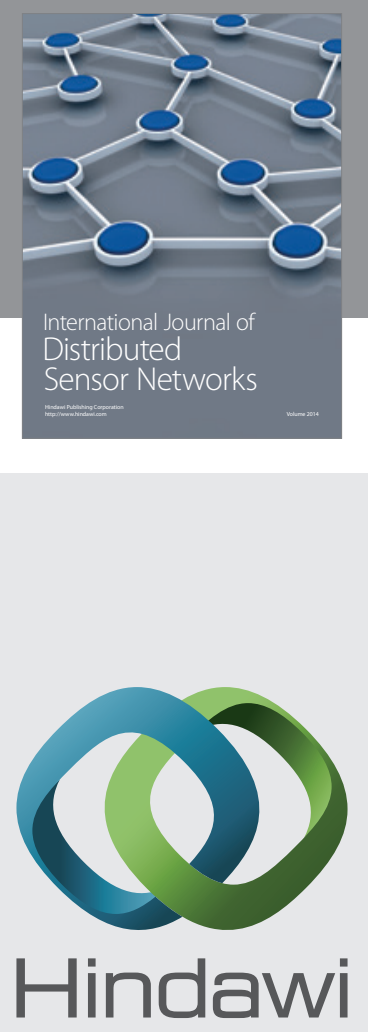

Submit your manuscripts at

http://www.hindawi.com
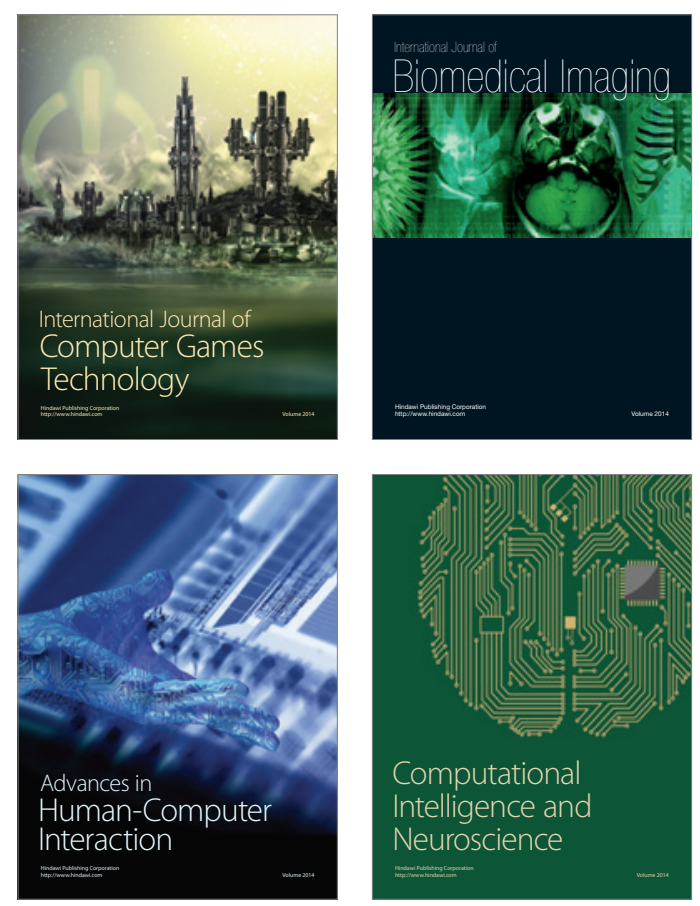
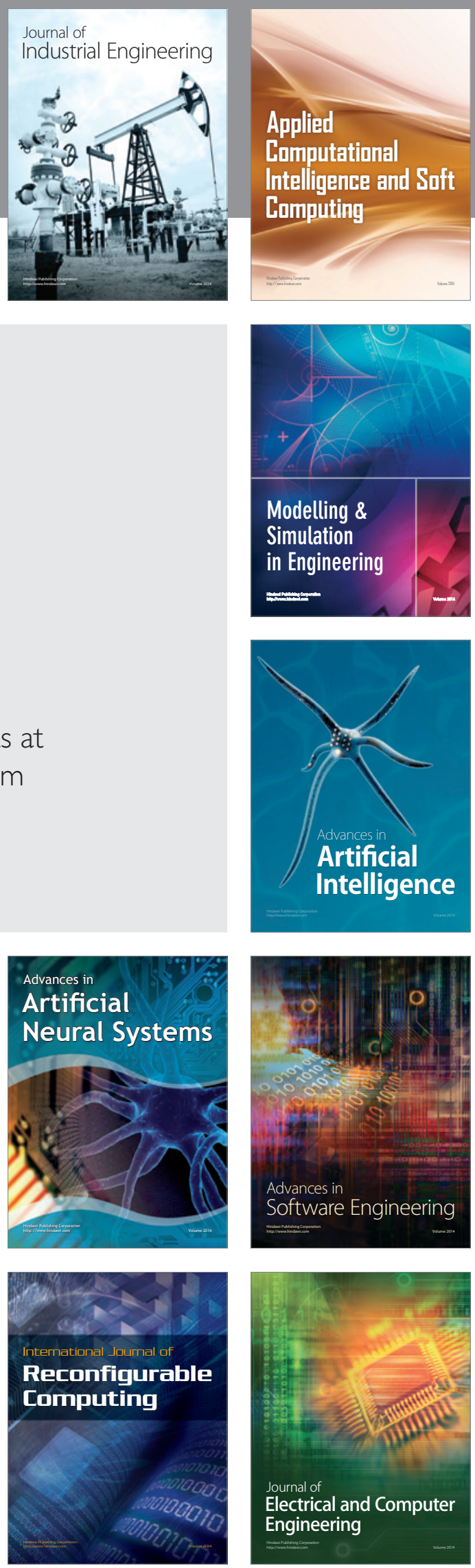